\title{
Electrospun Spider-net Structured Nanofibers Membrane from Homogeneous Solution of Nylon-6 and Poly (Ethylene oxide)
}

\author{
Manoj Kumar Jha ${ }^{1}$, Dinesh Shah ${ }^{1}$, Khuma Sharma Dhital' ${ }^{2}$, Lok Ranjan Bhatta ${ }^{3}$, Sahira Joshi ${ }^{1}$, \\ Ram Kumar Sharma ${ }^{1}$, Hem Raj Pant ${ }^{1 *}$ \\ ${ }^{1}$ Nano-Materials Lab, Department of Applied Sciences and Chemical Engineering, Pulchowk Campus, IOE, \\ Tribhuvan University, Kathmandu, Nepal \\ ${ }^{2}$ Department of Chemistry, Patan Multiple Campus, Tribhuvan University, Kathmandu, Nepal \\ ${ }^{3}$ Biological Resources Unit, Faculty of Science, Nepal Academy of Science \& Technology, \\ Khumaltar, Lalitpur, Nepal \\ *Corresponding E-mail: hempant@ioe.edu.np
}

(Received: Sept. 30, 2019; Revised: Dec. 23, 2019 \& Accepted: Dec. 24, 2019)

\begin{abstract}
Membrane development encompasses wide range of technology areas including process and product design, materials engineering, chemical engineering, as well as interaction phenomenon. Advances in membrane technology can solve most of the global concerns related to water, air, energy, healthcare and global warming. In this study, a simple electrospinning technique was applied to prepare composite nanofibers from Nylon-6 and polyethylene oxide (PEO) blend solutions. The effect of PEO on the morphology of the fiber was investigated. It was observed that the addition of PEO in the Nylon-6 solution resulted in the formation of ultrafine nanofibers along with the main fiber, which can be considered as a spider-net-like morphology. The nano/sub-nano arrangement of the fiber resembling a three-dimensional (3D) spider-net structure enhances the mechanical strength of the resulting nanofibers as compared to the pristine Nylon-6 nanofibers.
\end{abstract}

Keywords: Electrospinning, nylon-6/PEO composite, nanofiber, spider-net morphology

\section{Introduction}

In recent years, the development of electrospinning techniques has been increasingly investigated [1]. Electrospinning is considered as a simple and easy technique to fabricate the ultrafine continuous nanofibers morphology. Pure polymers, as well as the blending of a given polymer with other polymers/ oligomers, have been successfully electrospun into micro/ nanofiber matrices for various applications $[2,3]$. The fibers produced by this method have shown amazing characteristics, such as a very large surfaceto-volume ratio and a high porosity with a small pore size $[4,5]$. These characteristics make the electrospun fibers suitable for various potential applications such as filtration, protective clothing, tissue scaffold, wound dressing, drug delivery system, sensor, and optical materials [1,6-8].

The hybrid mats produced by electrospinning possess unique physical and chemical properties and therefore, research interest in the formation of polymer hybrid mats has been increased in the past decades. Despite the ease of nanofiber fabrication, it is yet challenging to fabricate electrospun nanofibers with a diameter of less than $10 \mathrm{~nm}$. After the successful preparation of nano-net like fibers via electrospinning process by Ding's group [9], benefits from the nano/subnano fibers have been realized and several research activities have been directed towards the development of nano-net like structures for various applications $[2,5,6,10]$. An important property of a polymer blend is the miscibility of its components because it affects the mechanical properties, the morphology, and the permeability and degradation [2]. Consequently, in this study, we report the preparation of a hybrid spider-web like a mat of polymer and oligomer using a simple electrospinning technique with the diameter of fibers ranging from nanometer to sub-nanometer 
scale. Nylon-6 is a biodegradable semi-crystalline polymeric material with good thermal, chemical, and mechanical properties [3,11]. Electrospinning of the blend solution of Nylon-6 and polyethylene oxide (PEO) may exhibit properties shown by both individual polymers and oligomer, thereby resulting in a material with improved properties. The choice of PEO is motivated by its wide variety of applications such as biomaterials including scaffolds, drug delivery [1], tissue engineering [12], wound healing, and conductive fibers.

\section{Materials and Methods}

Nylon-6 (medium molecular weight of KN 120 grade) was obtained from Kolon, Korea. Formic acid and acetic acid were purchased from Showa, Chemicals, Japan. Poly (ethylene oxide) oligomer was obtained from Sigma-Aldrich, USA. All the chemicals were used without any purification.

Nylon-6/PEO blend solutions were prepared by mixing at different content of PEO $(0.5 \%, 1 \%, 2 \%$, and $4 \%$ PEO with respect to the weight of Nylon-6) in Nylon-6 at 20\% final concentration. Formic acid and acetic acid (4:1) were used as the solvent. After stirring continuously to get a perfect blended solution, electrospinning was carried out. A high voltage power supply (CPS-60 K02 V1, Chungpa EMT, South Korea) of $22 \mathrm{kV}$ to the syringe micro-tip was supplied to electrospun the nanofibers. The tip-to-collector distance was kept at $16 \mathrm{~cm}$. The as-obtained nylon-6/ PEO nanofiber mats were vacuum dried in an oven at $30{ }^{\circ} \mathrm{C}$ for $12 \mathrm{~h}$ to remove the residual solvent and the samples were used for characterizations. The nanofiber mats containing $0.5,1,2$, and $4 \%$ PEO in Nylon-6 were denoted as NP0.5, NP1, NP2, and NP4, respectively. For comparison, pristine Nylon-6 nanofiber mat without using PEO is also prepared by the aforementioned process.

\section{Characterizations}

The morphology of the electrospun mats was investigated using FE-SEM (S-4700, Hitachi, Japan). Structural characterization was carried out by X-ray diffractometer (XRD, Rigaku, Japan) with $\mathrm{Cu} \mathrm{K}(\lambda=$ $1.540 \AA$ ) radiation over Bragg angles ranging from 10 to $80^{\circ}$. The bonding configurations of the samples were characterized by means of Fourier-transform infrared (FT-IR). Mechanical properties were measured with a universal testing machine (AG-5000G, Shimadzu,
Japan), under a crosshead speed of $10 \mathrm{~mm} / \mathrm{min}$. The samples were prepared in the form of a standard dumbbell-shaped according to ASTM Standard 638 via die-cutting from the mat and tested in the machine direction. Five samples were tested for each mat.

\section{Results and Discussion}

Figure 1 depicts the FE-SEM images of the assynthesized pristine Nylon-6 NFs and Nylon-6/ PEO composite NFs. As in figure 1A, the pristine Nylon-6 nanofibers exhibited a smooth, bead free, and continuous nanofibrous morphology throughout their lengths. The average diameter of the pristine Nylon-6 NFs was recorded as $213 \mathrm{~nm}$. In the case of composite nanofibers, a clear arrangement of spiderweb-like morphology due to the ultrafine nanofiber network was observed. It can be seen that the ultrafine nanofibers are connected to the main fiber. This type of spider-net-like arrangement of nanofibers provides a large surface area to volume ratio, thereby widening the applications of nanofibers. The average diameters of ultrafine in Mats NP0.5, NP1, NP2, and NP4 were found to be 21, 28, 32, $40 \mathrm{~nm}$, respectively.

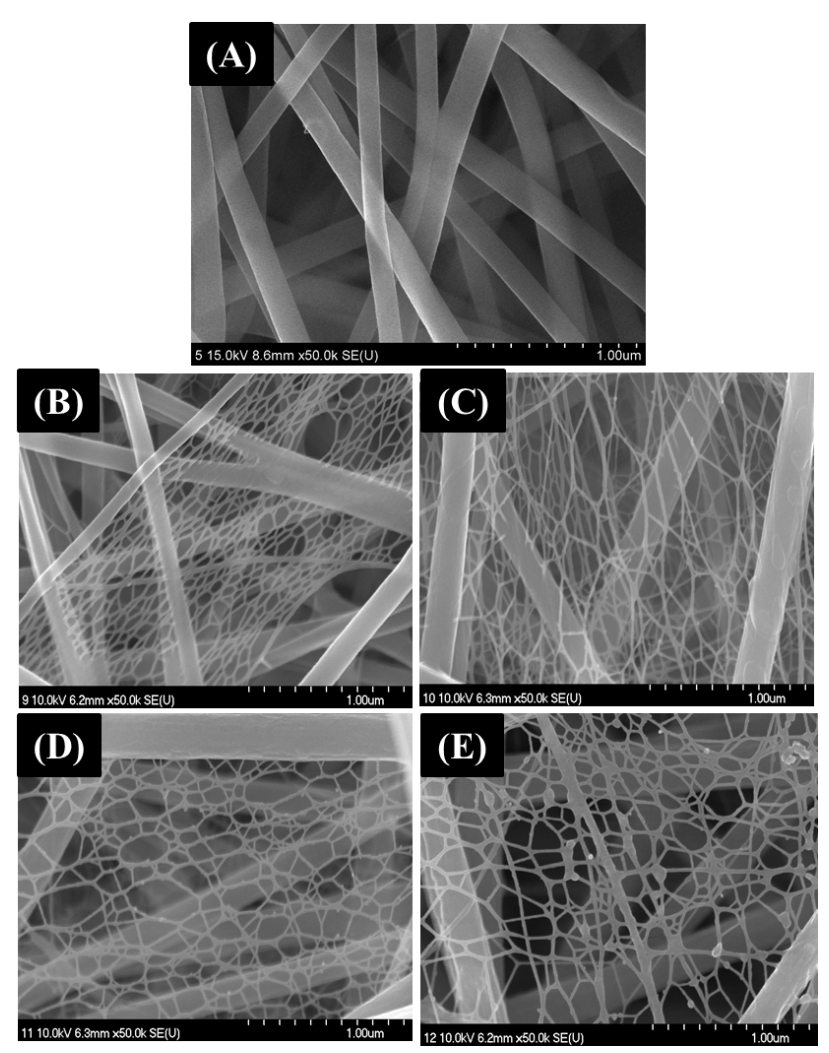

Figure 1: High magnification FESEM images of nanofibers: Nylon-6 (A), NP0.5 (B), NP1 (C), NP2 (D) and NP4 (E). 
Besides the variation in diameter, the arrangement of ultrafine nanofibers in the mats is different. The nanofibers in mat $\mathrm{N} 2$ show a three dimensional (3D) arrangement with multiple layers connecting to the main fiber body. At the lower content of PEO, the ultrafine nanofibers start to form, therefore they are distributed to the limited area (Figure 1B). With the increase in PEO content, a clear distribution of ultrafine fibers were observed; however, the pattern of arrangement is different. Among the four different samples, the best arrangement of spider-net fibers was observed in the NP2 sample (Figure 1C). Therefore, it can be concluded that $1 \%$ is the optimum content of PEO in the Nylon-6 solution for getting the nano/ sub-nano fibrous arrangement. The partial phase separation of homogeneously mixed higher molecular nylon and lower molecular PEO portion should be started from the stable jet region, and becomes more pronounced during vibrating and whipping of the jet during electrospinning [13]. During jet whipping, PEO forms a surface layer whereas nylon forms the core of the jet. From the surface PEO layer, sub-nano fibers (fiber diameter $<50 \mathrm{~nm}$ ) are formed whereas from core nylon, nanofibers (fiber diameter $>200 \mathrm{~nm}$ ) are formed.

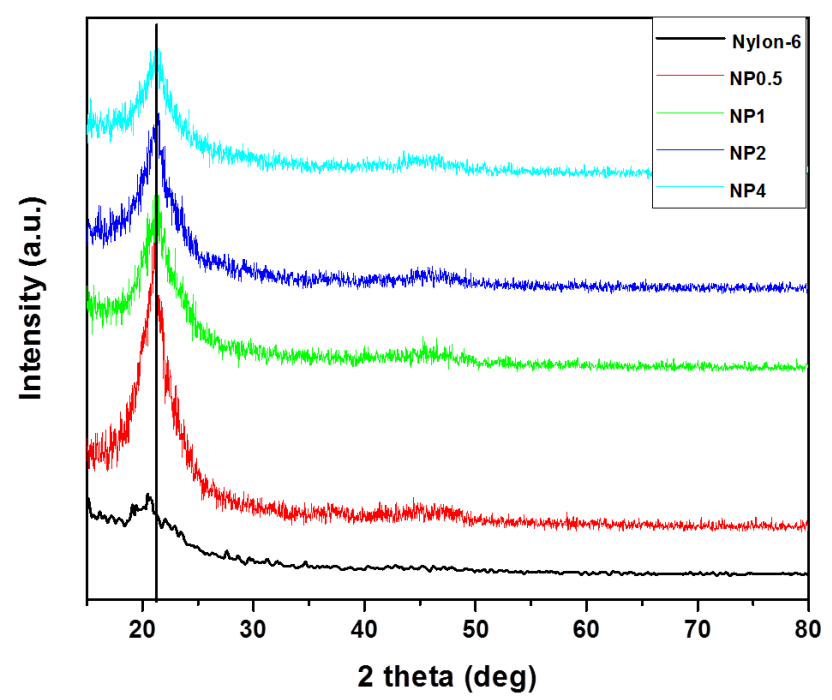

Figure 2: XRD spectra of different formulations.

XRD analysis was carried out in order to examine the crystal structure of the prepared samples. Nylon-6 nanofiber exhibited a characteristic peak at approximately $2 \theta$ values of $20.5^{\circ}$, which can be termed as $\alpha$ crystalline phase [13]. After blending with PEO, the diffraction peak was observed at approximately
$2 \theta$ values of $21.2^{\circ}$. This shifting towards the higher values of $2 \theta^{\circ}$ indicated a conversion from $\alpha$ to $\gamma$ phase [13].

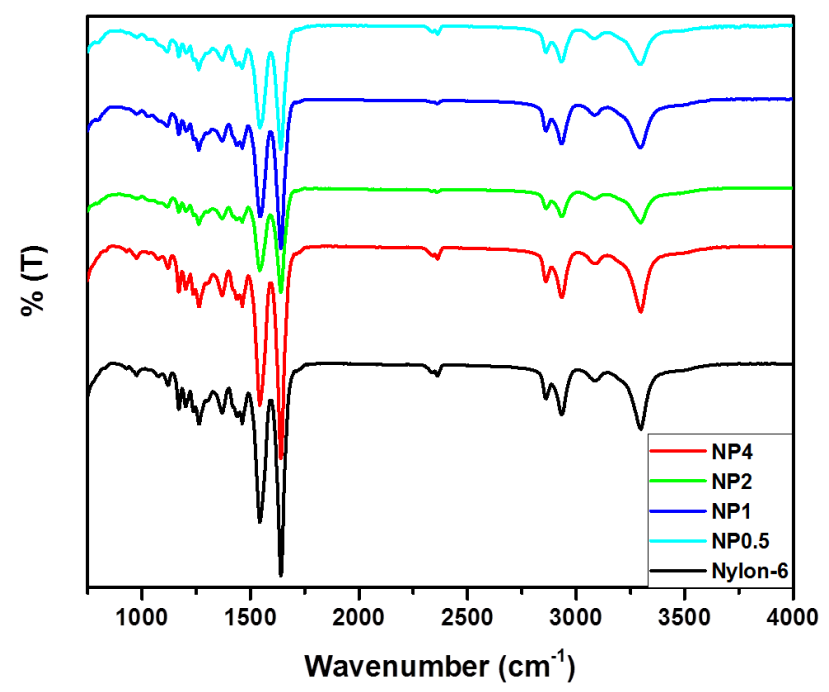

Figure 3: IR spectra of Nylon-6 and Nylon-6/PEO composite nanofibers.

Figure 3 depicts the characteristic FTIR peaks of pristine nylon-6 and nylon-6/PEO blend nanofiber composites. In pristine Nylon- 6 nanofibers, the bands appeared at 3300 and $3085 \mathrm{~cm}^{-1}$ correspond to the NH stretching vibrations [14]. The peaks at 2860 and $2930 \mathrm{~cm}^{-1}$ belong to the $\mathrm{C}-\mathrm{H}$ stretching vibrations. The bands at 1640 and $1445 \mathrm{~cm}^{-1}$ are associated with $(\mathrm{C}=\mathrm{O})$ amide $\mathrm{I}$ and $(\mathrm{C}-\mathrm{N})$ amide $\mathrm{II}$, respectively $[14,15]$. These bands were observed to be decreased

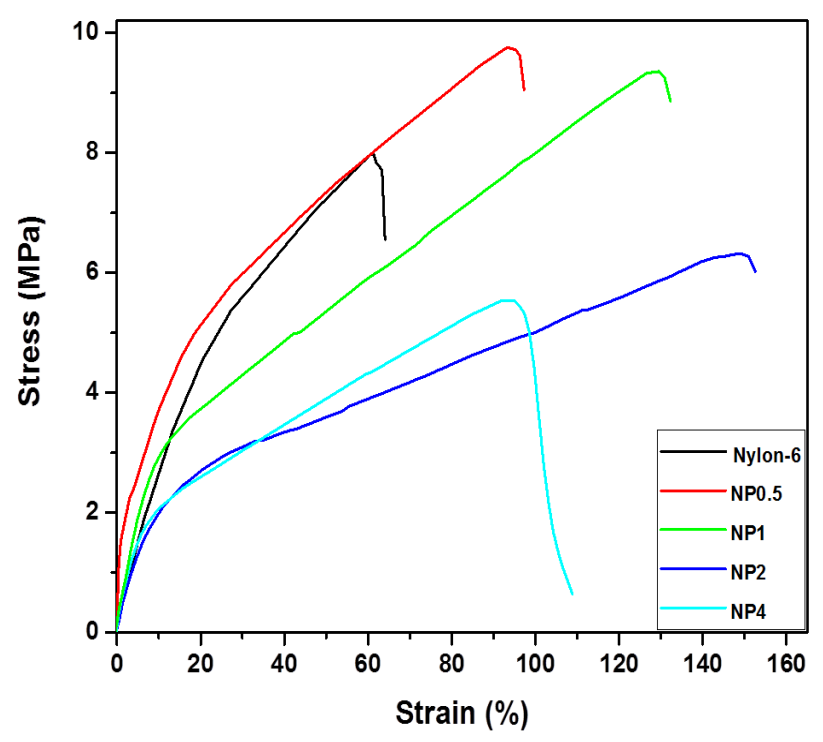

Figure 4: Stress-strain curves of various formulations of nanofiber mats. 
in the case of composite nanofibers, which may be due to the hydrogen bonding with PEO. We studied the mechanical properties of the as-synthesized mats by the UTM machine. The study revealed that at the lower content of PEO, the mechanical property of the Nylon-6 is increased, whereas with further increasing PEO content, the mechanical strength decreased. The highest mechanical performance in the case of the NP1 sample is attributed to the well-formed 3D spider-net like morphology. As compared to the other samples, the sub-nano fibers are highly interconnected with the main fibers in NP1. The FESEM morphologies (Figure 1) also support the mechanical strength data.

\section{Conclusion}

Nylon-6 and PEO polymers are properly blended with each other to produce composite bimodal electrospun nanofibrous membranes. 3D spider-net morphology of Nylon-6/PEO composite nanofibers was successfully prepared by the electrospinning technique. The interconnected fibrous morphology improved the mechanical property of the hybrid fibers. Thus prepared high aspect ratio Nylon-6/PEO composite nanofibers can be used in different fields such as filtration, wound healing, etc.

\section{Acknowledgments}

The research work was supported by the World Academy of Science, Trieste, Italy (TWAS)/Swedish International Development Corporation Agency (Sida) (project number; 18-168RG/ CHE/AS_GFR3240303651) and University Grant Commission, Nepal (project number; -CRG-74/74Engg-02). We are thankful to Chonbuk National University, the Republic of Korea for characterizing the samples.

\section{References}

1. B. Pant, M. Park and S-J. Park, Drug delivery applications of core-sheath nanofibers prepared by coaxial electrospinning: a review, Pharmaceutics 2019, 11, 305.

2. H. R. Pant, M. P. Bajgai, K. T. Nam, K. H. Chu, K. H. Park and H. Y. Park, Formation of electrospun nylon-6/methoxy poly(ethyleneglycol) oligomer spider-wave nanofibers, Materials Letters, 2010, 64, 2087-2090. (doi:doi.10.1016/j.matlet. 2010.06.047).

3. B. Pant, H. R. Pant, D. R. Pandeya, G. Panthi,
K. T. Nam, S. T. Hong, C. S. Kim and H. Y. Kim, Characterization and antibacterial properties of Ag NPs loaded nylon-6 nanocomposite prepared by one-step electrospinning process, Colloids and Surfaces A: Physicochemical and Engineering Aspects, 2012, 395, 94-99. (doi:doi.10.1016/j. colsurfa.2011.12.011).

4. B. Pant, M. Park, S-J. Park and H. Y. Kim, High strength electrospun nanofiber mats via CNT reinforcement: A review, Composites Research, 2016, 29, 186-193. (doi:10.7234/ composres.2016.29.4.186).

5. X. Wang, B. Ding, G. Sun, M. Wang and J. Yu, Electro-spinning/netting: A strategy for the fabrication of three-dimensional polymer nano-fiber/nets, Progress in Materials Science, 2013, 58, 1173-1243. (doi:doi.10.1016/j. pmatsci.2013.05.001).

6. B. Pant, M. Park and S-J. Park, One-step synthesis of silver nanoparticles embedded polyurethane nano-fiber/net structured membrane as an effective antibacterial medium, Polymers 2019, 11, 1185 .

7. L. Ping and D. Bin, Applications of electrospun fibers, Recent Patents on Nanotechnology 2008, 2, 169-182. (doi:d oi.10.2174/187221008786369688).

8. H. R. Pant, B. Pant, P. Pokharel, H. J. Kim, L. D. Tijing, C. H. Park, D. S. Lee, H. Y. Kim and C. S. Kim, Photocatalytic $\mathrm{TiO}_{2}-\mathrm{RGO} /$ nylon-6 spiderwave-like nano-nets via electrospinning and hydrothermal treatment, Journal of Membrane Science, 2013, 429, 225-234. (doi:doi.10.1016/j. memsci.2012.11.025).

9 B. Ding, Li. C. Y. Miyauchi, O. Kuwaki and S. Shiratori, Formation of novel 2D polymer nanowebs via electrospinning, Nanotechnolog, 2006, 17, 3685-3691. (doi:10.1088/09574484/17/15/011).

10. H. R. Pant, H. J. Kim, M. K. Joshi, B. Pant, C. H. Park, J. I. Kim, K. S. Hui and C. S. Kim, Onestep fabrication of multifunctional composite polyurethane spider-web-like nanofibrous membrane for water purification, Journal of Hazardous Materials, 2014, 264, 25-33. (doi:doi.10.1016/j.jhazmat.2013.10.066). 
11. H. R. Pant, P. Risal, C. H. Park , L. D. Tijing, Y. J. Jeong and C. S. Kim, Core-shell structured electrospun biomimetic composite nanofibers of calcium lactate/nylon-6 for tissue engineering, Chemical Engineering Journal, 2013, 221, 90-98. (doi:doi.10.1016/j.cej.2013.01.072).

12. P. Basu, A. Repanas, A. Chatterjee, B. Glasmacher, U. N. Kumar and I. Manjubala, PEO-CMC blend nanofibers fabrication by electrospinning for soft tissue engineering applications, Materials Letters, 2017, 195, 10-13. (doi:doi.10.1016/j. matlet.2017.02.065).
13. Y. C. Tseng and S-P. Rwei, Synthesis and characterization of the feed ratio of polyethylene oxide (0 10 wt \% PEO) in the nylon-6/PEO copolymer system, Journal of Applied Polymer Science, 2012, 123, 796-806. (doi:10.1002/ app.34653).

14. K-H. Lee, K-W. Kim, A. Pesapane, H-Y. Kim and J. F. Rabolt, Polarized FT-IR study of macroscopically oriented electrospun nylon-6 nanofibers, Macromolecules, 2008, 41, 14941498. (doi:10.1021/ma701927w). 